DOI 10.7603/s40956-015-0006-5

Moroccan J. Pure and Appl. Anal.(MJPAA)

RESEARCH ARTICLE

Volume 1(2), 2015, Pages 76-90

ISSN: $2351-8227$

\title{
On a Fourth Order Parabolic Equation with Mixed Type Boundary Conditions in a Nonrectangular Domain
}

\author{
Arezki Kheloufi ${ }^{a}$
}

ABstract. This paper is devoted to the study of the following fourth order parabolic equation $\partial_{t} u+\partial_{x}^{4} u=f$ in the non-necessarily rectangular domain

$$
Q=\left\{(t, x) \in \mathbb{R}^{2}: 0<t<T, \varphi_{1}(t)<x<\varphi_{2}(t)\right\} .
$$

The equation is subject to mixed type conditions $\partial_{x} u=\partial_{x}^{3} u+\beta u=0$, on the lateral boundary of $Q$. The right-hand side term $f$ of the equation lies in $L_{\omega}^{2}(Q)$ the space of square-integrable functions on $Q$ with the measure $\omega d t d x$. Our aim is to find sufficient conditions on the coefficient $\beta$ and on the functions $\varphi_{i}, i=1,2$ and on the weight $\omega$ such that the solution of this equation belongs to the anisotropic Sobolev space

$$
H_{\omega}^{1,4}(Q)=\left\{u \in L_{\omega}^{2}(Q): \partial_{t} u, \partial_{x}^{j} u \in L_{\omega}^{2}(Q), j=1,2,3,4\right\} .
$$

The analysis is performed by using the domain decomposition method.

2010 Mathematics Subject Classification. 35K05,35K55.

Key words and phrases. Higher-order parabolic equations, Nonregular domains, Anisotropic weighted Sobolev spaces, Mixed type conditions.

\section{Introduction}

Let $Q$ be an open set of $\mathbb{R}^{2}$ defined by

$$
Q=\left\{(t, x) \in \mathbb{R}^{2}: 0<t<T ; \varphi_{1}(t)<x<\varphi_{2}(t)\right\},
$$

Received April 21, 2015 - Accepted July 03, 2015.

(C)The Author(s) 2015. This article is published with open access by Sidi Mohamed Ben Abdallah University

${ }^{a}$ Laboratoire des Mathématiques Appliquées, Faculté des Sciences Exactes, Université de Bejaia, 6000 Béjaia, Algeria Lab. E.D.P.N.L., Ecole Normale Supérieure, 16050-Kouba, Algiers, Algeria

e-mail: arezkinet2000@yahoo.fr 
where $T$ is a finite positive number, while $\varphi_{1}$ and $\varphi_{2}$ are continuous real-valued functions defined on $[0, T]$, Lipschitz continuous on $[0, T]$, and such that

$$
\varphi(t):=\varphi_{2}(t)-\varphi_{1}(t)>0
$$

for $t \in] 0, T]$. The lateral boundary of $Q$ is defined by

$$
\Gamma_{i}=\left\{\left(t, \varphi_{i}(t)\right) \in \mathbb{R}^{2}: 0<t<T\right\}, i=1,2 .
$$

We will then assume that

$$
\begin{gathered}
\varphi(0)=0, \\
\varphi_{i}^{\prime}(t) \varphi^{2}(t) \rightarrow 0 \quad \text { as } t \rightarrow 0, \quad i=1,2 .
\end{gathered}
$$

In $Q$, we consider the mixed type boundary value problem

$$
\left\{\begin{array}{l}
\partial_{t} u+\partial_{x}^{4} u=f \text { in } Q, \\
\left.\partial_{x} u\right|_{\Gamma_{i}}=\partial_{x}^{3} u+\left.\beta_{i} u\right|_{\Gamma_{i}}=0, i=1,2 .
\end{array}\right.
$$

The right-hand side term $f$ of the equation lies in $L_{\omega}^{2}(Q)$ the space of square-integrable functions on $Q$ with the measure $\omega d t d x$. Here the weight $\omega$ is a real-valued function defined on $[0, T]$, differentiable on $] 0, T]$ and such that

$$
\forall t \in[0, T]: \omega(t)>0 \text {. }
$$

We also assume that

$$
\omega \text { is a decreasing function on }] 0, T] \text {. }
$$

The coefficients $\beta_{i}, i=1,2$ are real numbers such that

$$
\begin{gathered}
\beta_{1}>0 \text { and } \beta_{2}<0 \\
\left.(-1)^{i+1}\left(\beta_{i}+\frac{\varphi_{i}^{\prime}(t)}{2}\right) \geq 0 \text { a.e. } t \in\right] 0, T[, i=1,2 .
\end{gathered}
$$

Note that such kind of boundary conditions

$$
\left.\partial_{x} u\right|_{\Gamma_{i}}=\partial_{x}^{3} u+\left.\beta_{i} u\right|_{\Gamma_{i}}=0, i=1,2,
$$

can be found in [1] where the existence of classical solutions to a one-dimensional nonlinear fourth-order elliptic equation arising in quantum semi-conductor modeling was proved. These specific boundary conditions are important for the originality of this work. Indeed, to my knowledge, results concerning higher-order parabolic equations on time-varying domains, subject to such kind of boundary conditions, have not appeared in the litterature to date.

It is well known that there are two main approaches for the study of boundary value problems in such non-smooth domains. We can work directly in the non-regular domains and we obtain singular solutions (see, for example [8] and [12]). Some authors have considered the singulariries which appear in the solutions when the domain is nonregular, as Kondratiev in [11] and Sadallah in [19] and [20]. Or, we impose conditions on the non-regular domains to obtain regular solutions (see, for example [9] and [18]). It is the second approach that we follow in this work. So, under the above mentioned conditions on the functions $\varphi_{1}, \varphi_{2}$ and $\omega$ and on the coefficients $\beta_{i}, i=1,2$, we will 
prove that Problem (3) has a solution with optimal regularity, that is a solution $u$ belonging to the anisotropic weighted Sobolev space

$$
H_{\gamma, \omega}^{1,4}(Q)=\left\{u \in H_{\omega}^{1,4}(Q):\left.\partial_{x} u\right|_{\Gamma_{i}}=\partial_{x}^{3} u+\left.\beta_{i} u\right|_{\Gamma_{i}}=0, i=1,2\right\},
$$

with

$$
H_{\omega}^{1,4}(Q)=\left\{u \in L_{\omega}^{2}(Q): \partial_{t} u, \partial_{x}^{j} u \in L_{\omega}^{2}(Q), j=1, \ldots, 4\right\} .
$$

The solvability of boundary value problems for a $2 m$-th order parabolic equation in Hölder spaces for noncylindrical domains (of the same kind but which can not include our domain) with a nonsmooth (in t) lateral boundary was established in [3], [5] and [6]. In Sadallah [18] the same problem has been studied for a $2 \mathrm{~m}$-parabolic operator in the case of Cauchy-Dirichlet boundary conditions. Further references on the analysis of higher-order parabolic problems in noncylindrical domains are: Galaktionov [7], Baderko [2], Kheloufi [10] Labbas and Sadallah [13], Mikhailov [16] and [17].

Observe that the domain $Q$ considered here is nonstandard since it shrinks at $t=0$, $(\varphi(0)=0)$. So, in Section 3, we prove that Problem (3) admits a (unique) solution when $Q$ could be transformed into a regular domain by means of a regular change of variable, i.e., we suppose that $\varphi_{1}(0)<\varphi_{2}(0)$. In Section 4 we approximate $Q$ by a sequence $\left(Q_{n}\right)$ of such domains and we establish (for $T$ small enough) a uniform estimate of the type

$$
\left\|u_{n}\right\|_{H_{\omega}^{1,4}\left(Q_{n}\right)} \leq K\|f\|_{L_{\omega}^{2}\left(Q_{n}\right)},
$$

where $u_{n}$ is the solution of Problem (3) in $Q_{n}$ and $K$ is a constant independent of $n$. This allows us to take limits in $\left(Q_{n}\right)$ in order to reach the domain $Q$. In the following section, we give some results we need to develop further arguments.

\section{Preliminaries}

\subsection{Uniqueness of solutions.}

Theorem 2.1. Assume that the weight function $\omega$ verifies assumptions (4) and (5). Then, under the condition (7) on the coefficients $\beta_{i}, i=1,2$, and on the functions of parametrization $\varphi_{i}, i=1,2$, the solution of Problem (3) is unique.

Proof. Let us consider $u \in H_{\gamma, \omega}^{1,4}(Q)$ a solution of the problem (3) with a null right-hand side term. So,

$$
\partial_{t} u+\partial_{x}^{4} u=0 \text { in } Q
$$

In addition $u$ fulfils the boundary conditions

$$
\left.\partial_{x} u\right|_{\Gamma_{i}}=\partial_{x}^{3} u+\left.\beta_{i} u\right|_{\Gamma_{i}}=0, i=1,2 .
$$

Using Green formula, we have

$$
\begin{aligned}
\int_{Q}\left(\partial_{t} u+\partial_{x}^{4} u\right) u \cdot \omega(t) d t d x= & \int_{\partial Q}\left[\frac{1}{2}|u|^{2} \nu_{t}+\left(\partial_{x}^{3} u \cdot u-\partial_{x}^{2} u . \partial_{x} u\right) \nu_{x}\right] \omega(t) d \sigma \\
& +\int_{Q}\left|\partial_{x}^{2} u\right|^{2} \omega(t) d t d x-\int_{Q} \frac{1}{2}|u|^{2} \omega^{\prime}(t) d t d x,
\end{aligned}
$$

where $\nu_{t}$ and $\nu_{x}$ are the components of the unit outward normal vector at $\partial Q$. We shall rewrite the boundary integral making use of the boundary conditions. On the part of 
the boundary where $t=T$, we have $\nu_{x}=0$ and $\nu_{t}=1$. Accordingly the corresponding boundary integral

$$
\frac{1}{2} \int_{\varphi_{1}(T)}^{\varphi_{2}(T)}|u|^{2}(T, x) \omega(T) d x
$$

is nonnegative. On the part of the boundary where $x=\varphi_{i}(t), i=1,2$, we have

$$
\nu_{x}=\frac{(-1)^{i}}{\sqrt{1+\left(\varphi_{i}^{\prime}\right)^{2}(t)}}, \nu_{t}=\frac{(-1)^{i+1} \varphi_{i}^{\prime}(t)}{\sqrt{1+\left(\varphi_{i}^{\prime}\right)^{2}(t)}}
$$

and

$$
\partial_{x} u\left(t, \varphi_{i}(t)\right)=\partial_{x}^{3} u\left(t, \varphi_{i}(t)\right)+\beta_{i} u\left(t, \varphi_{i}(t)\right)=0, i=1,2 .
$$

Consequently the corresponding boundary integral is

$$
\int_{0}^{T}\left(\frac{\varphi_{1}^{\prime}(t)}{2}+\beta_{1}\right) u^{2}\left(t, \varphi_{1}(t)\right) \omega(t) d t-\int_{0}^{T}\left(\frac{\varphi_{2}^{\prime}(t)}{2}+\beta_{2}\right) u^{2}\left(t, \varphi_{2}(t)\right) \omega(t) d t .
$$

Then, we obtain

$$
\begin{aligned}
& \int_{Q}\left(\partial_{t} u+\partial_{x}^{4} u\right) u \omega(t) d t d x \\
& =\sum_{i=1}^{2} \int_{0}^{T}(-1)^{i+1}\left(\beta_{i}+\frac{\varphi_{i}^{\prime}(t)}{2}\right) u^{2}\left(t, \varphi_{i}(t)\right) \omega(t) d t \\
& +\frac{1}{2} \int_{\varphi_{1}(T)}^{\varphi_{2}(T)} u^{2}(T, x) \omega(t) d x+\int_{Q}\left|\partial_{x}^{2} u\right|^{2} \omega(t) d t d x-\int_{Q} \frac{1}{2}|u|^{2} \omega^{\prime}(t) d t d x .
\end{aligned}
$$

Consequently

$$
\int_{Q}\left(\partial_{t} u+\partial_{x}^{4} u\right) u \omega(t) d t d x=0
$$

yields

$$
\int_{Q}\left(\left|\partial_{x}^{2} u\right|^{2}\right) \omega(t) d t d x=0
$$

because

$$
\int_{\varphi_{1}(T)}^{\varphi_{2}(T)} \frac{1}{2}|u|^{2} \omega(T) d x-\int_{Q} \frac{1}{2}|u|^{2} \omega^{\prime}(t) d t d x \geq 0
$$

thanks to the conditions (4) and (5) and

$$
\sum_{i=1}^{2} \int_{0}^{T}(-1)^{i+1}\left(\beta_{i}+\frac{\varphi_{i}^{\prime}(t)}{2}\right) u^{2}\left(t, \varphi_{i}(t)\right) \omega(t) d t \geq 0
$$

thanks to the condition (7). This implies that $\left|\partial_{x}^{2} u\right|^{2}=0$ and consequently $\partial_{x}^{4} u=0$. Then, the hypothesis $\partial_{t} u+\partial_{x}^{4} u=0$ gives $\partial_{t} u=0$. Thus, $u$ is constant. The boundary conditions and the fact that $\beta_{i} \neq 0, i=1,2$ imply that $u=0$ in $Q$. This proves the uniqueness of the solution of Problem (3). 


\subsection{Technical Lemmas.}

Lemma 2.1. Assume that $\beta_{i}, i=1,2$ fulfil the condition (6). Then, there exists a positive constant $K_{1}$ such that for each $u \in H_{\gamma}^{4}(0,1)$

$$
\left\|u^{(k)}\right\|_{L^{2}(0,1)} \leq K_{1}\left\|u^{(4)}\right\|_{L^{2}(0,1)}, k=0,1,2,3
$$

where

$$
H_{\gamma}^{4}(0,1)=\left\{u \in H^{4}(0,1): u^{(3)}(j)+\beta_{j+1} u(j)=u^{(1)}(j)=0, j=0,1\right\} .
$$

Here, $u^{(k)}, k=1,2,3,4$ is the derivative of order $k$ of $u$ on $(0,1)$ and $u^{(0)}=u$.

Proof. Let $f$ an arbitrary fixed element of $L^{2}(0,1)$. Every solution of the ordinary differential equation $u^{(4)}=f$ is of the form

$$
u(y)=\int_{0}^{y} \int_{0}^{x} \int_{0}^{w} \int_{0}^{t} f(s) d s d t d w d x+\frac{1}{6} u^{(3)}(0) y^{3}+\frac{1}{2} u^{(2)}(0) y^{2}+u^{(1)}(0) y+u(0), y \in[0,1] .
$$

The variables $u^{(k)}(0), k=0,1,2,3$, are to be determined in a unique way such that the boundary conditions $u^{(3)}(j)+\beta_{j+1} u(j)=u^{(1)}(j)=0, j=0,1$ are satisfied.

From the preceding representation of the solution and thus also its derivatives

$$
\begin{gathered}
u^{(3)}(y)=\int_{0}^{y} f(s) d s+u^{(3)}(0), y \in[0,1], \\
u^{(2)}(y)=\int_{0}^{y} \int_{0}^{x} f(s) d s d x+u^{(3)}(0) y+u^{(2)}(0), y \in[0,1], \\
u^{(1)}(y)=\int_{0}^{y} \int_{0}^{x} \int_{0}^{w} f(s) d s d w d x+\frac{1}{2} u^{(3)}(0) y^{2}+u^{(2)}(0) y+u^{(1)}(0), y \in[0,1],
\end{gathered}
$$

and from the required mixed boundary conditions we obtain the following system to be solved:

$$
\left\{\begin{array}{l}
u^{(3)}(0)+0 u^{(2)}(0)+\beta_{1} u(0)=0 \\
\left(1+\frac{\beta_{2}}{6}\right) u^{(3)}(0)+\frac{\beta_{2}}{2} u^{(2)}(0)+\beta_{2} u(0)=-\int_{0}^{1} f(s) d s-\beta_{2} \int_{0}^{1} \int_{0}^{x} \int_{0}^{w} \int_{0}^{t} f(s) d s d t d w d x \\
\frac{1}{2} u^{(3)}(0)+u^{(2)}(0)+0 u(0)=-\int_{0}^{1} \int_{0}^{x} \int_{0}^{w} f(s) d s d w d x .
\end{array}\right.
$$

This system in the unknowns $u^{(k)}(0), k=0,2,3$, is uniquely solvable if and only if

$$
-\beta_{2}+\beta_{1}\left(1-\frac{\beta_{2}}{6}\right) \neq 0 .
$$

This condition is verified thanks to (6). Finally, the unique solution of the problem

$$
\left\{\begin{array}{l}
u^{(4)}=f \\
u^{(3)}(j)+\beta_{j+1} u(j)=0, j=0,1, \\
u^{(1)}(j)=0, j=0,1
\end{array}\right.
$$

is given by

$$
u(y)=\int_{0}^{y} \int_{0}^{x} \int_{0}^{w} \int_{0}^{t} f(s) d s d t d w d x+\frac{1}{6} u^{(3)}(0) y^{3}+\frac{1}{2} u^{(2)}(0) y^{2}+u(0), y \in[0,1],
$$


where

$$
\begin{aligned}
u(0)= & \frac{\int_{0}^{1} f(s) d s+\beta_{2} \int_{0}^{1} \int_{0}^{x} \int_{0}^{w} \int_{0}^{t} f(s) d s d t d w d x-\frac{\beta_{2}}{2} \int_{0}^{1} \int_{0}^{x} \int_{0}^{w} f(s) d s d w d x}{-\beta_{2}+\beta_{1}\left(1-\frac{\beta_{2}}{6}\right)}, \\
u^{(2)}(0)= & \frac{\beta_{2}-\beta_{1}\left(1+\frac{\beta_{2}}{6}\right) \int_{0}^{1} \int_{0}^{x} \int_{0}^{w} f(s) d s d w d x}{-\beta_{2}+\beta_{1}\left(1-\frac{\beta_{2}}{6}\right)} \\
& +\frac{\beta_{1}\left[\frac{1}{2} \int_{0}^{1} f(s) d s+\frac{\beta_{2}}{2} \int_{0}^{1} \int_{0}^{x} \int_{0}^{w} \int_{0}^{t} f(s) d s d t d w d x\right]}{-\beta_{2}+\beta_{1}\left(1-\frac{\beta_{2}}{6}\right)}, \\
u^{(3)}(0)= & \frac{\beta_{1}\left[-\int_{0}^{1} f(s) d s-\beta_{2} \int_{0}^{1} \int_{0}^{x} \int_{0}^{w} \int_{0}^{t} f(s) d s d t d w d x+\frac{\beta_{2}}{2} \int_{0}^{1} \int_{0}^{x} \int_{0}^{w} f(s) d s d w d x\right]}{-\beta_{2}+\beta_{1}\left(1-\frac{\beta_{2}}{6}\right)} .
\end{aligned}
$$

Using the Cauchy-Schwarz inequality, we obtain the following estimates

$$
\left|u^{(k)}(0)\right| \leq C\|f\|_{L^{2}(0,1)}, k=0,2,3
$$

which will allow us to obtain the desired estimates.

Lemma 2.2. Under the assumption (6) on $\beta_{i}, i=1,2$, there exists a positive constant $C_{1}$ (independent of $a$ and $b$ ) such that

$$
\left\|v^{(k)}\right\|_{L^{2}(a, b)}^{2} \leq C_{1}(b-a)^{2(4-k)}\left\|v^{(4)}\right\|_{L^{2}(a, b)}^{2}, k=0,1,2,3
$$

for each $v \in H_{\gamma}^{4}(a, b)$, with

$$
H_{\gamma}^{4}(a, b)=\left\{v \in H^{4}(a, b): \begin{array}{l}
v^{(3)}(a)+\frac{\beta_{1}}{(b-a)^{3}} v(a)=v^{(3)}(b)+\frac{\beta_{2}}{(b-a)^{3}} v(b)=0, \\
v^{(1)}(a)=v^{(1)}(b)=0
\end{array}\right\} .
$$

Proof. It is a direct consequence of Lemma 2.1. Indeed, we define the following affine change of variable

$$
[0,1] \longrightarrow[a, b], x \mapsto(1-x) a+x b=y .
$$

and we set $u(x)=v(y)$. Then if $u \in H_{\gamma}^{4}(0,1), v$ belongs to $H_{\gamma}^{4}(a, b)$. We have

$$
\begin{aligned}
\left\|u^{(l)}\right\|_{L^{2}(0,1)}^{2} & =\int_{0}^{1}\left(u^{(l)}\right)^{2}(x) d x \\
& =\int_{a}^{b}\left(v^{(l)}\right)^{2}(y)(b-a)^{2 l} \frac{d y}{b-a} \\
& =\int_{a}^{b}\left(v^{(l)}\right)^{2}(y)(b-a)^{2 l-1} d y \\
& =(b-a)^{2 l-1}\left\|v^{(l)}\right\|_{L^{2}(a, b)}^{2}
\end{aligned}
$$

where $l \in\{0,1,2,3\}$. On the other hand, we have

$$
\begin{aligned}
\left\|u^{(4)}\right\|_{L^{2}(0,1)}^{2} & =\int_{0}^{1}\left(u^{(4)}\right)^{2}(x) d x \\
& =\int_{a}^{b}\left(v^{(4)}\right)^{2}(y)(b-a)^{7} d y \\
& =(b-a)^{7}\left\|v^{(4)}\right\|_{L^{2}(a, b)}^{2}
\end{aligned}
$$


Using the inequality

$$
\left\|u^{(l)}\right\|_{L^{2}(0,1)}^{2} \leq K_{1}\left\|u^{(4)}\right\|_{L^{2}(0,1)}^{2}
$$

of Lemma 2.1, we obtain the desired inequality

$$
\left\|v^{(l)}\right\|_{L^{2}(] a, b[)}^{2} \leq(b-a)^{2(4-l)} K_{2}\left\|v^{(4)}\right\|_{L^{2}(] a, b[)}^{2}, l=0,1,2,3,
$$

with $K_{2}=K_{1}$.

Remark 2.1. In Lemmas 2.1 and 2.2 we can replace $\|\cdot\|_{L^{2}}$ by $\|\cdot\|_{L_{\omega}^{2}}$.

\section{Resolution of Problem (3) in a "model" domain $Q_{n}$}

In this section, we replace $Q$ by

$$
Q_{n}=\left\{(t, x) \in Q: \frac{1}{n}<t<T\right\},
$$

with $n \in \mathbb{N}^{*}$. Thus, $\varphi_{1}\left(\frac{1}{n}\right)<\varphi_{2}\left(\frac{1}{n}\right)$ and we have

Theorem 3.1. For each $n \in \mathbb{N}^{*}$ such that $\frac{1}{n}<T$, the problem

$$
\left\{\begin{array}{l}
\partial_{t} u_{n}+\partial_{x}^{4} u_{n}=f_{n} \in L_{\omega}^{2}\left(Q_{n}\right), \\
\left.u_{n}\right|_{t=\frac{1}{n}}=0, \\
\left.\partial_{x} u_{n}\right|_{\Gamma_{i, n}}=\partial_{x}^{3} u_{n}+\left.\beta_{i} u_{n}\right|_{\Gamma_{i, n}}=0, i=1,2,
\end{array}\right.
$$

where $\Gamma_{i, n}=\left\{(t, x) \in \Gamma_{i}: \frac{1}{n}<t<T\right\}, i=1,2$, admits a (unique) solution $u_{n} \in$ $H_{\omega}^{1,4}\left(Q_{n}\right)$.

Proof. The uniqueness of the solution is easy to check, thanks to the boundary conditions. Let us prove its existence. The change of variable

$$
(t, x) \longmapsto(t, y)=\left(t, \frac{x-\varphi_{1}(t)}{\varphi(t)}\right),
$$

transforms $Q_{n}$ into the rectangle $\left.P_{n}=\right] \frac{1}{n}, T[\times] 0,1\left[\right.$. Putting $f_{n}(t, x)=g_{n}(t, y)$ and $u_{n}(t, x)=v_{n}(t, y)$, then Problem (8) becomes

$$
\left\{\begin{array}{l}
\partial_{t} v_{n}+a(t, y) \partial_{y} v_{n}+c(t) \partial_{y}^{4} v_{n}=g_{n} \in L_{\omega}^{2}\left(P_{n}\right), \\
\left.v_{n}\right|_{t=\frac{1}{n}}=0 \\
\left.\partial_{y} v_{n}\right|_{\Gamma_{i, P_{n}}}=\partial_{y}^{3} v_{n}+\left.\beta_{i} \varphi^{3}(t) v_{n}\right|_{\Gamma_{i, P_{n}}}=0, i=1,2,
\end{array}\right.
$$

where $\left.\Gamma_{1, P_{n}}=\right] \frac{1}{n}, T\left[\times\{0\}, \Gamma_{2, P_{n}}=\right] \frac{1}{n}, T\left[\times\{1\}, c(t)=\frac{1}{\varphi^{4}(t)}\right.$ and $a(t, y)=-\frac{y \varphi^{\prime}(t)+\varphi_{1}^{\prime}(t)}{\varphi(t)}$.

Since the functions $a, c$ and $\varphi$ are bounded when $t \in] \frac{1}{n}, T[$, then the above change of variable which is bi-Lipschitz preserves the spaces $L_{\omega}^{2}$ and $H_{\omega}^{1,4}$. In other words

$$
f_{n} \in L_{\omega}^{2}\left(Q_{n}\right) \Longleftrightarrow g_{n} \in L_{\omega}^{2}\left(P_{n}\right), u_{n} \in H_{\omega}^{1,4}\left(Q_{n}\right) \Longleftrightarrow v_{n} \in H_{\omega}^{1,4}\left(P_{n}\right) \text {. }
$$


Theorem 3.2. For each $n \in \mathbb{N}^{*}$ such that $\frac{1}{n}<T$, the operator

$$
L^{\prime}=\partial_{t}+a \partial_{y}+c \partial_{y}^{4}
$$

is an isomorphism from $H_{\gamma, \omega}^{1,4}\left(P_{n}\right)$ into $L_{\omega}^{2}\left(P_{n}\right)$, with

$$
H_{\gamma, \omega}^{1,4}\left(P_{n}\right)=\left\{v_{n} \in H_{\omega}^{1,4}\left(P_{n}\right):\left.v_{n}\right|_{t=\frac{1}{n}}=\left.\partial_{y} v_{n}\right|_{\Gamma_{i, P_{n}}}=\partial_{y}^{3} v_{n}+\left.\beta_{i} \varphi^{3}(t) v_{n}\right|_{\Gamma_{i, P_{n}}}=0, i=1,2\right\} .
$$

Consider the simplified problem

$$
\left\{\begin{array}{l}
\partial_{t} v_{n}+c(t) \partial_{y}^{4} v_{n}=g_{n} \in L_{\omega}^{2}\left(P_{n}\right), \\
\left.v_{n}\right|_{t=\frac{1}{n}}=0 \\
\left.\partial_{y} v_{n}\right|_{\Gamma_{i, P_{n}}}=\partial_{y}^{3} v_{n}+\left.\beta_{i} \varphi^{3}(t) v_{n}\right|_{\Gamma_{i, P_{n}}}=0, i=1,2 .
\end{array}\right.
$$

Note that $g_{n} \in L_{\omega}^{2}\left(P_{n}\right)$ if and only if $f_{n} \in L_{\omega}^{2}\left(Q_{n}\right)$.

Lemma 3.1. For every $g_{n} \in L_{\omega}^{2}\left(P_{n}\right)$, there exists a unique $v_{n} \in H_{\gamma, \omega}^{1,4}\left(P_{n}\right)$ solution of (9).

Proof. Since the coefficient $c(t)$ is continuous in $\overline{P_{n}}$, the optimal regularity is given by Ladyzhenskaya-Solonnikov-Ural'tseva [14].

Lemma 3.2. For each $n \in \mathbb{N}^{*}$ such that $\frac{1}{n}<T$, the following operator is compact

$$
B: H_{\gamma, \omega}^{1,4}\left(P_{n}\right) \longrightarrow L_{\omega}^{2}\left(P_{n}\right), v_{n} \mapsto B v_{n}=a(t, y) \partial_{y} v_{n}
$$

Proof. $P_{n}$ has the "horn property" of Besov [4], so

$$
\partial_{y}: H_{\gamma, \omega}^{1,4}\left(P_{n}\right) \longrightarrow H_{\omega}^{\frac{3}{4}, 3}\left(P_{n}\right), v_{n} \mapsto \partial_{y} v_{n}
$$

is continuous. Since $P_{n}$ is bounded, the canonical injection is compact from $H_{\omega}^{\frac{3}{4}, 3}\left(P_{n}\right)$ into $L_{\omega}^{2}\left(P_{n}\right)$, where

$$
H^{\frac{3}{4}, 3}\left(P_{n}\right)=L^{2}\left(\frac{1}{n}, T ; H^{3}(] 0,1[)\right) \cap H^{\frac{3}{4}}\left(\frac{1}{n}, T ; L^{2}(] 0,1[)\right) .
$$

For the complete definitions of the $H^{r, s}$ Hilbertian Sobolev spaces see for instance [15]. Consider the composition

$$
\partial_{y}: H_{\gamma, \omega}^{1,4}\left(P_{n}\right) \longrightarrow H_{\omega}^{\frac{3}{4}, 3}\left(P_{n}\right) \longrightarrow L_{\omega}^{2}\left(P_{n}\right), v_{n} \mapsto \partial_{y} v_{n} \mapsto \partial_{y} v_{n}
$$

then $\partial_{y}$ is a compact operator from $H_{\gamma, \omega}^{1,4}\left(P_{n}\right)$ into $L_{\omega}^{2}\left(P_{n}\right)$. Since $a(.,$.$) is a bounded$ function, the operator $a \partial_{y}$ is also compact from $H_{\gamma, \omega}^{1,4}\left(P_{n}\right)$ into $L_{\omega}^{2}\left(P_{n}\right)$.

Lemma 3.1 shows that the operator $L_{1}=\partial_{t}+c \partial_{y}^{4}$ is an isomorphism from $H_{\gamma, \omega}^{1,4}\left(P_{n}\right)$ into $L_{\omega}^{2}\left(P_{n}\right)$; on the other hand the operator $a \partial_{y}$ is compact, consequently, $L_{1}+a \partial_{y}$ is a Fredholm operator from $H_{\gamma, \omega}^{1,4}\left(P_{n}\right)$ into $L_{\omega}^{2}\left(P_{n}\right)$. Thus the invertibility of $L_{1}+a \partial_{y}$ follows from its injectivity. 
Lemma 3.3. For each $n \in \mathbb{N}^{*}$ such that $\frac{1}{n}<T$, the space $\left\{v_{n} \in D\left(\left[\frac{1}{n}, T\right] ; H^{4}(0,1)\right):\left.v_{n}\right|_{t=\frac{1}{n}}=\left.\partial_{y} v_{n}\right|_{\Gamma_{i, P_{n}}}=\partial_{y}^{3} v_{n}+\left.\beta_{i} \varphi^{3}(t) v_{n}\right|_{\Gamma_{i, P_{n}}}=0, i=1,2\right\}$,

is dense in

$H_{\gamma}^{1,4}\left(P_{n}\right)=\left\{v_{n} \in H^{1,4}\left(P_{n}\right):\left.v_{n}\right|_{t=\frac{1}{n}}=\left.\partial_{y} v_{n}\right|_{\Gamma_{i, P_{n}}}=\partial_{y}^{3} v_{n}+\left.\beta_{i} \varphi^{3}(t) v_{n}\right|_{\Gamma_{i, P_{n}}}=0, i=1,2\right\}$.

It is a particular case of Theorem 2.1 [15].

We shall need the following result in order to justify some calculations in this section.

Lemma 3.4. For each $n \in \mathbb{N}^{*}$ such that $\frac{1}{n}<T$, the space

$$
\left\{v_{n} \in H^{4}\left(P_{n}\right):\left.v_{n}\right|_{t=\frac{1}{n}}=\left.\partial_{y} v_{n}\right|_{\Gamma_{i, P_{n}}}=\partial_{y}^{3} v_{n}+\left.\beta_{i} \varphi^{3}(t) v_{n}\right|_{\Gamma_{i, P_{n}}}=0, i=1,2\right\},
$$

is dense in the space

$$
\left\{v_{n} \in H^{1,4}\left(P_{n}\right):\left.v_{n}\right|_{t=\frac{1}{n}}=\left.\partial_{y} v_{n}\right|_{\Gamma_{i, P_{n}}}=\partial_{y}^{3} v_{n}+\left.\beta_{i} \varphi^{3}(t) v_{n}\right|_{\Gamma_{i, P_{n}}}=0, i=1,2\right\} .
$$

Remark 3.1. In Lemma 3.4, we can replace $P_{n}$ by $Q_{n}$ with the help of the change of variable defined above.

\section{Resolution of Problem (3) in a "small" triangular domain}

4.1. A uniform estimate. Now, we return to the nonrectangular domain $Q$. For each $n \in \mathbb{N}^{*}$ such that $\frac{1}{n}<T$, we denote $f_{n}=\left.f\right|_{Q_{n}}$ and $u_{n} \in H_{\omega}^{1,4}\left(Q_{n}\right)$ the solution of Problem (8) in $Q_{n}$. Such a solution exists by Theorem 3.1.

Proposition 4.1. For $T$ small enough, there exists a constant $M$ independent of $n$ such that

$$
\left\|u_{n}\right\|_{H_{\omega}^{1,4}\left(Q_{n}\right)} \leq M\left\|f_{n}\right\|_{L_{\omega}^{2}\left(Q_{n}\right)} \leq M\|f\|_{L_{\omega}^{2}(Q)} .
$$

Remark 4.1. Let $\epsilon>0$ be a real which we will choose small enough. The hypothesis (2) implies the existence of a real number $T>0$ small enough such that

$$
\forall t \in(0, T),\left|\varphi^{\prime}(t) \varphi^{2}(t)\right| \leq \epsilon .
$$

In order to prove Proposition 4.1, we need the following lemmas.

Lemma 4.1. For every $\epsilon>0$, chosen such that $\varphi(t) \leq \epsilon$, there exists a constant $C_{1}$ independent of $n$ such that

$$
\left\|\partial_{x}^{l} u_{n}\right\|_{L_{\omega}^{2}\left(Q_{n}\right)}^{2} \leq C_{1} \epsilon^{2(4-l)}\left\|\partial_{x}^{4} u_{n}\right\|_{L_{\omega}^{2}\left(Q_{n}\right)}^{2}, l=0,1,2,3 .
$$

Proof. Replacing in Lemma $2.2 u$ by $u_{n}$ and $] a, b[$ by $] \varphi_{1}(t), \varphi_{2}(t)[$, for a fixed $t$, we obtain

$$
\begin{aligned}
\int_{\varphi_{1}(t)}^{\varphi_{2}(t)}\left(\partial_{x}^{l} u_{n}\right)^{2} d x & \leq K_{2}(\varphi(t))^{2(4-l)} \int_{\varphi_{1}(t)}^{\varphi_{2}(t)}\left(\partial_{x}^{4} u_{n}\right)^{2} d x \\
& \leq K_{2} \epsilon^{2(4-l)} \int_{\varphi_{1}(t)}^{\varphi_{2}(t)}\left(\partial_{x}^{4} u_{n}\right)^{2} d x .
\end{aligned}
$$

Multiplying the previous inequality by $\omega(t)$ (which is positive) and integrating with respect to $t$, we get the desired result with $C_{1}=K_{2}$. 
Lemma 4.2. Let us denote the inner product in $L_{\omega}^{2}\left(Q_{n}\right)$ by $\langle.,$.$\rangle , then we have$

$$
2\left\langle\partial_{t} u_{n}, \partial_{x}^{4} u_{n}\right\rangle \geq-K \epsilon\left\|\partial_{x}^{4} u_{n}\right\|_{L_{\omega}^{2}\left(Q_{n}\right)} .
$$

Proof. We have

$$
\partial_{t} u_{n} . \partial_{x}^{4} u_{n}=\partial_{x}\left(\partial_{t} u_{n} \cdot \partial_{x}^{3} u_{n}\right)-\partial_{x}\left(\partial_{x} \partial_{t} u_{n} \cdot \partial_{x}^{2} u_{n}\right)+\frac{1}{2} \partial_{t}\left(\partial_{x}^{2} u_{n}\right)^{2}
$$

Then

$$
\begin{aligned}
2\left\langle\partial_{t} u_{n}, \partial_{x}^{4} u_{n}\right\rangle= & 2 \int_{Q_{n}} \partial_{t} u_{n} \cdot \partial_{x}^{4} u_{n} \cdot \omega(t) d t d x \\
= & 2 \int_{Q_{n}} \partial_{x}\left(\partial_{t} u_{n} \cdot \partial_{x}^{3} u_{n}\right) \cdot \omega(t) d t d x+\int_{Q_{n}} \partial_{t}\left(\partial_{x}^{2} u_{n}\right)^{2} \cdot \omega(t) d t d x \\
& -2 \int_{Q_{n}} \partial_{x}\left(\partial_{x} \partial_{t} u_{n} \cdot \partial_{x}^{2} u_{n}\right) \cdot \omega(t) d t d x \\
= & \int_{\partial Q_{n}}\left[\left(\partial_{x}^{2} u_{n}\right)^{2} \nu_{t}+2\left(\partial_{t} u_{n} \cdot \partial_{x}^{3} u_{n}-\partial_{x} \partial_{t} u_{n} \cdot \partial_{x}^{2} u_{n}\right) \nu_{x}\right] \cdot \omega(t) d \sigma \\
& -\int_{Q_{n}}\left(\partial_{x}^{2} u_{n}\right)^{2} \cdot \omega^{\prime}(t) d t d x .
\end{aligned}
$$

We shall rewrite the boundary integral making use of the boundary conditions. On the part of the boundary of $Q_{n}$ where $t=\frac{1}{n}$, we have $\partial_{x} u_{n}=0$ and consequently $\partial_{x}^{2} u_{n}=\partial_{x}^{3} u_{n}=0$. The corresponding boundary integral vanishes. On the part of the boundary where $t=T$, we have $\nu_{x}=0$ and $\nu_{t}=1$. Accordingly the corresponding boundary integral

$$
\int_{\varphi_{1}(T)}^{\varphi_{2}(T)}\left[\partial_{x}^{2} u_{n}(T, x)\right]^{2} \omega(T) d x
$$

is nonnegative. On the parts of the boundary where $x=\varphi_{i}(t), i=1,2$, we have

$$
\nu_{x}=\frac{(-1)^{i}}{\sqrt{1+\left(\varphi_{i}^{\prime}\right)^{2}(t)}}, \nu_{t}=\frac{(-1)^{i+1} \varphi_{i}^{\prime}(t)}{\sqrt{1+\left(\varphi_{i}^{\prime}\right)^{2}(t)}}
$$

and

$$
\partial_{x} u_{n}=\partial_{x}^{3} u_{n}+\beta_{i} u_{n}=0, i=1,2 .
$$

Differentiating with respect to $t$, we obtain

$$
\partial_{t} \partial_{x} u_{n}\left(t, \varphi_{i}(t)\right)=-\varphi_{i}^{\prime}(t) \partial_{x}^{2} u_{n}\left(t, \varphi_{i}(t)\right), i=1,2 .
$$

Consequently, the corresponding boundary integrals $I_{n, k}$ and $J_{n, k}, k=1,2$ are the following:

$$
\begin{aligned}
I_{n, k} & =(-1)^{k} \int_{\frac{1}{n}}^{T} \varphi_{k}^{\prime}(t)\left[\partial_{x}^{2} u_{n}\left(t, \varphi_{k}(t)\right)\right]^{2} d t, k=1,2 \\
J_{n, k} & =(-1)^{k+1} 2 \int_{\frac{1}{n}}^{T} \beta_{k} \partial_{t} u_{n}\left(t, \varphi_{k}(t)\right) \cdot u_{n}\left(t, \varphi_{k}(t)\right) d t, k=1,2 .
\end{aligned}
$$

In virtue of (4) and (5), we have

$$
2\left\langle\partial_{t} u_{n}, \partial_{x}^{4} u_{n}\right\rangle \geq-\left|I_{n, 1}\right|-\left|I_{n, 2}\right|-\left|J_{n, 1}\right|-\left|J_{n, 2}\right|
$$


Lemma 4.3. There exists a constant $K_{3}$ independent of $n$ such that

$$
\left|I_{n, k}\right| \leq K_{3} \epsilon\left\|\partial_{x}^{4} u_{n}\right\|_{L_{\omega}^{2}\left(Q_{n}\right)}^{2}, \quad k=1,2 .
$$

Proof. We convert the boundary integral $I_{n, 1}$ into a surface integral by setting

$$
\begin{aligned}
{\left[\partial_{x}^{2} u_{n}\left(t, \varphi_{1}(t)\right)\right]^{2}=} & -\left.\frac{\varphi_{2}(t)-x}{\varphi(t)}\left[\partial_{x}^{2} u_{n}(t, x)\right]^{2}\right|_{x=\varphi_{1}(t)} ^{x=\varphi_{2}(t)} \\
= & -\int_{\varphi_{1}(t)}^{\varphi_{2}(t)} \partial_{x}\left\{\frac{\varphi_{2}(t)-x}{\varphi(t)}\left[\partial_{x}^{2} u_{n}\right]^{2}\right\} d x \\
= & -2 \int_{\varphi_{1}(t)}^{\varphi_{2}(t)} \frac{\varphi_{2}(t)-x}{\varphi(t)} \partial_{x}^{2} u_{n} . \partial_{x}^{3} u_{n} d x \\
& +\int_{\varphi_{1}(t)}^{\varphi_{2}(t)} \frac{1}{\varphi(t)}\left[\partial_{x}^{2} u_{n}\right]^{2} d x .
\end{aligned}
$$

Then, we have

$$
\begin{aligned}
I_{n, 1}= & -\int_{\frac{1}{n}}^{T} \varphi_{1}^{\prime}(t)\left[\partial_{x}^{2} u_{n}\left(t, \varphi_{1}(t)\right)\right]^{2} \omega(t) d t \\
= & -\int_{Q_{n}} \frac{\varphi_{1}^{\prime}(t)}{\varphi(t)}\left[\partial_{x}^{2} u_{n}(t, x)\right]^{2} \omega(t) d t d x \\
& +2 \int_{Q_{n}} \frac{\varphi_{2}(t)-x}{\varphi(t)} \varphi_{1}^{\prime}(t)\left(\partial_{x}^{2} u_{n}\right)\left(\partial_{x}^{3} u_{n}\right) \omega(t) d t d x .
\end{aligned}
$$

Thanks to Lemma 2.2, we can write

$$
\int_{\varphi_{1}(t)}^{\varphi_{2}(t)}\left[\partial_{x}^{2} u_{n}\right]^{2} d x \leq K_{2}[\varphi(t)]^{4} \int_{\varphi_{1}(t)}^{\varphi_{2}(t)}\left[\partial_{x}^{4} u_{n}\right]^{2} d x
$$

Therefore

$$
\int_{\varphi_{1}(t)}^{\varphi_{2}(t)}\left[\partial_{x}^{2} u_{n}\right]^{2} \frac{\left|\varphi_{1}^{\prime}\right|}{\varphi} \omega(t) d x \leq K_{2}\left|\varphi_{1}^{\prime}\right|[\varphi(t)]^{3} \int_{\varphi_{1}(t)}^{\varphi_{2}(t)}\left[\partial_{x}^{4} u_{n}\right]^{2} \omega(t) d x
$$

consequently

$$
\begin{aligned}
\left|I_{n, 1}\right| \leq & K_{2} \int_{Q_{n}}\left|\varphi_{1}^{\prime}\right|[\varphi(t)]^{3}\left(\partial_{x}^{4} u_{n}\right)^{2} \omega(t) d t d x \\
& +2 \int_{Q_{n}}\left|\varphi_{1}^{\prime}\right|\left|\partial_{x}^{2} u_{n}\right|\left|\partial_{x}^{3} u_{n}\right| \omega(t) d t d x
\end{aligned}
$$

since $\left|\frac{\varphi_{2}(t)-x}{\varphi(t)}\right| \leq 1$. Using the inequality

$$
2\left|\varphi_{1}^{\prime} \partial_{x}^{2} u_{n}\right|\left|\partial_{x}^{3} u_{n}\right| \leq \epsilon\left(\partial_{x}^{3} u_{n}\right)^{2}+\frac{1}{\epsilon}\left(\varphi_{1}^{\prime}\right)^{2}\left(\partial_{x}^{2} u_{n}\right)^{2}
$$

for all $\epsilon>0$, we obtain

$$
\begin{aligned}
\left|I_{n, 1}\right| \leq & K_{2} \int_{Q_{n}}\left|\varphi_{1}^{\prime}\right|[\varphi]^{3}\left(\partial_{x}^{4} u_{n}\right)^{2} \omega(t) d t d x \\
& +\int_{Q_{n}} \epsilon\left(\partial_{x}^{3} u_{n}\right)^{2} \omega(t) d t d x+\frac{1}{\epsilon} \int_{Q_{n}}\left(\varphi_{1}^{\prime}\right)^{2}\left(\partial_{x}^{2} u_{n}\right)^{2} \omega(t) d t d x
\end{aligned}
$$

Lemma 4.1 yields

$$
\frac{1}{\epsilon} \int_{Q_{n}}\left(\varphi_{1}^{\prime}\right)^{2}\left(\partial_{x}^{2} u_{n}\right)^{2} \omega(t) d t d x \leq K_{2} \frac{1}{\epsilon} \int_{Q_{n}}\left(\varphi_{1}^{\prime}\right)^{2}[\varphi]^{4}\left(\partial_{x_{1}}^{4} u_{n}\right)^{2} \omega(t) d t d x
$$

Thus,

$$
\begin{aligned}
\left|I_{n, 1}\right| \leq & K_{2} \int_{Q_{n}}\left[\left|\varphi_{1}^{\prime}\right|[\varphi]^{3}+\frac{1}{\epsilon}\left(\varphi_{1}^{\prime}\right)^{2}[\varphi]^{4}\right]\left(\partial_{x}^{4} u_{n}\right)^{2} \omega(t) d t d x \\
& +\int_{Q_{n}} \epsilon\left(\partial_{x}^{3} u_{n}\right)^{2} \omega(t) d t d x \\
\leq & \left(K_{2}+1\right) \epsilon \int_{Q_{n}}\left(\partial_{x}^{4} u_{n}\right)^{2} \omega(t) d t d x
\end{aligned}
$$

since $\left|\varphi_{1}^{\prime} \varphi^{2}\left(\varphi-\varphi_{1}^{\prime} \varphi^{2}\right)\right| \leq \epsilon$. Finally, taking $K_{3}=\left(K_{2}+1\right)$, we obtain

$$
\left|I_{n, 1}\right| \leq K_{3} \epsilon\left\|\partial_{x}^{4} u_{n}\right\|_{L_{\omega}^{2}\left(Q_{n}\right)} .
$$


The inequality

$$
\left|I_{n, 2}\right| \leq K_{3} \epsilon\left\|\partial_{x}^{4} u_{n}\right\|_{L_{\omega}^{2}\left(Q_{n}\right)},
$$

can be proved by a similar argument.

2. Estimation of $J_{n, k}, k=1,2$ : We have

$$
\begin{aligned}
J_{n, 1} & =2 \int_{\frac{1}{n}}^{T} \beta_{1} \partial_{t} u_{n}\left(t, \varphi_{1}(t)\right) \cdot u_{n}\left(t, \varphi_{1}(t)\right) d t \\
& =\int_{\frac{1}{n}}^{T} \beta_{1}\left[\partial_{t} u_{n}^{2}\left(t, \varphi_{1}(t)\right)\right] d t .
\end{aligned}
$$

By setting

$$
h(t)=u_{n}^{2}\left(t, \varphi_{1}(t)\right),
$$

we obtain

$$
\begin{aligned}
J_{n, 1} & =\int_{\frac{1}{n}}^{T} \beta_{1} \cdot\left[h^{\prime}(t)-\varphi_{1}^{\prime}(t) \partial_{x} u_{n}^{2}\left(t, \varphi_{1}(t)\right)\right] d t \\
& \left.=\beta_{1} \cdot h(t)\right]_{\frac{1}{n}}^{T}-\int_{\frac{1}{n}}^{T} \beta_{1} \cdot \varphi_{1}^{\prime}(t) \partial_{x} u_{n}^{2}\left(t, \varphi_{1}(t)\right) d t
\end{aligned}
$$

Thanks to (6) and the fact that $u_{n}^{2}\left(\frac{1}{n}, \varphi_{1}\left(\frac{1}{n}\right)\right)=0$, we have

$$
\left.\beta_{1} \cdot h(t)\right]_{\frac{1}{n}}^{T} \geq 0 \text {. }
$$

The last boundary integral in the expression of $J_{n, 1}$ can be treated by a similar argument used in Lemma 4.3. So, we obtain the existence of a positive constant $K$ independent of $n$, such that

$$
\left|\int_{\frac{1}{n}}^{T} \beta_{1} \cdot \varphi_{1}^{\prime}(t) \partial_{x} u_{n}^{2}\left(t, \varphi_{1}(t)\right) d t\right| \leq K \epsilon\left\|\partial_{x}^{4} u_{n}\right\|_{L^{2}\left(Q_{n}\right)}^{2} .
$$

By a similar method, we obtain

$$
\left.J_{n, 2}=-\beta_{2} u_{n}^{2}\left(t, \varphi_{2}(t)\right)\right]_{\frac{1}{n}}^{T}+\int_{\frac{1}{n}}^{T} \beta_{2} \cdot \varphi_{2}^{\prime}(t) \partial_{x} u_{n}^{2}\left(t, \varphi_{2}(t)\right) d t
$$

Thanks to (6) and the fact that $u_{n}^{2}\left(\frac{1}{n}, \varphi_{2}\left(\frac{1}{n}\right)\right)=0$, we have

$$
\left.\beta_{2} u_{n}^{2}\left(t, \varphi_{2}(t)\right)\right]_{\frac{1}{n}}^{T} \geq 0
$$

Then

$$
\left|\int_{\frac{1}{n}}^{T} \beta_{2} \cdot \varphi_{2}^{\prime}(t) \partial_{x} u_{n}^{2}\left(t, \varphi_{2}(t)\right) d t\right| \leq K \epsilon\left\|\partial_{x}^{4} u_{n}\right\|_{L^{2}\left(Q_{n}\right)}^{2}
$$

where $K$ is a positive constant independent of $n$.

Proof of Proposition (4.1): We have

$$
\begin{aligned}
\left\|f_{n}\right\|_{L_{\omega}^{2}\left(Q_{n}\right)}^{2} & =\left\langle\partial_{t} u_{n}+\partial_{x}^{4} u_{n}, \partial_{t} u_{n}+\partial_{x}^{4} u_{n}\right\rangle \\
& =\left\|\partial_{t} u_{n}\right\|_{L_{\omega}^{2}\left(Q_{n}\right)}^{2}+\left\|\partial_{x}^{4} u_{n}\right\|_{L_{\omega}^{2}\left(Q_{n}\right)}^{2}+2\left\langle\partial_{t} u_{n}, \partial_{x}^{4} u_{n}\right\rangle .
\end{aligned}
$$


Summing up the estimates (11), (12) and (13) of the inner products and making use of Lemma 4.3, we then obtain

$$
\begin{aligned}
\left\|f_{n}\right\|_{L_{\omega}^{2}\left(Q_{n}\right)}^{2} & \geq\left\|\partial_{t} u_{n}\right\|_{L_{\omega}^{2}\left(Q_{n}\right)}^{2}+\left\|\partial_{x}^{4} u_{n}\right\|_{L_{\omega}^{2}\left(Q_{n}\right)}^{2}-\left|I_{n, 1}\right|-\left|I_{n, 2}\right|-\left|J_{n, 1}\right|-\left|J_{n, 2}\right| \\
& \geq\left\|\partial_{t} u_{n}\right\|_{L_{\omega}^{2}\left(Q_{n}\right)}^{2}+(1-N \epsilon)\left\|\partial_{x}^{4} u_{n}\right\|_{L^{2}\left(Q_{n}\right)}^{2},
\end{aligned}
$$

where $N$ is a constant independent of $n$. Then, it is sufficient to choose $\epsilon$ such that $(1-N \epsilon)>0$ to get a constant $K_{0}>0$ independent of $n$ such that

$$
\left\|f_{n}\right\|_{L_{\omega}^{2}\left(Q_{n}\right)} \geq K_{0}\left\|u_{n}\right\|_{H_{\omega}^{1,4}\left(Q_{n}\right)}
$$

and since

$$
\left\|f_{n}\right\|_{L_{\omega}^{2}\left(Q_{n}\right)} \leq\|f\|_{L_{\omega}^{2}(Q)}
$$

there exists a constant $M>0$, independent of $n$ satisfying

$$
\left\|u_{n}\right\|_{H_{\omega}^{1,4}\left(Q_{n}\right)} \leq M\left\|f_{n}\right\|_{L_{\omega}^{2}\left(Q_{n}\right)} \leq M\|f\|_{L_{\omega}^{2}(Q)} .
$$

This completes the proof of Proposition (4.1).

\subsection{Passing to the limit.}

Theorem 4.1. Assume that $\beta_{1}, \beta_{2}, \varphi_{1}$ and $\varphi_{2}$ fulfil conditions (6), (7), (1) and (2) and the weight function $\omega$ verifies assumptions (4) and (5). Then for $T$ small enough, the fourth order parabolic operator

$$
L=\partial_{t}+\partial_{x}^{4}
$$

is an isomorphism from $H_{\gamma, \omega}^{1,4}(Q)$ into $L_{\omega}^{2}(Q)$.

Proof. Choose a sequence $\left(Q_{n}\right)_{n \in \mathbb{N}^{*}}$ of the domains defined above (see Section 3), such that $Q_{n} \subseteq Q$. Then, we have $Q_{n} \rightarrow Q$, as $n \rightarrow \infty$. Consider the solution $u_{n} \in H_{\omega}^{1,4}\left(Q_{n}\right)$ of the mixed problem

$$
\left\{\begin{array}{l}
\partial_{t} u_{n}+\partial_{x}^{4} u_{n}=f_{n} \in L_{\omega}^{2}\left(Q_{n}\right), \\
\left.u_{n}\right|_{t=\frac{1}{n}}=0 \\
\left.\partial_{x} u_{n}\right|_{\Gamma_{i, n}}=\partial_{x}^{3} u_{n}+\left.\beta_{i} u_{n}\right|_{\Gamma_{i, n}}=0, i=1,2,
\end{array}\right.
$$

where $\Gamma_{i, n}=\left\{(t, x) \in \Gamma_{i}: \frac{1}{n}<t<T\right\}, i=1,2$. Such a solution $u_{n}$ exists by Theorem 3.1. Let $\widetilde{u_{n}}$ the 0 -extension of $u_{n}$ to $Q$. In virtue of Proposition 4.1, we know that there exists a constant $C$ such that

$$
\left\|\widetilde{u_{n}}\right\|_{L_{\omega}^{2}(Q)}+\left\|\widetilde{\partial_{t} u_{n}}\right\|_{L_{\omega}^{2}(Q)}+\sum_{j=1}^{4}\left\|\widetilde{\partial_{x}^{j} u_{n}}\right\|_{L_{\omega}^{2}(Q)} \leq C\|f\|_{L_{\omega}^{2}(Q)} .
$$

This means that $\widetilde{u_{n}}, \widetilde{\partial_{t} u_{n}}, \widetilde{\partial_{x}^{j} u_{n}}$ for $j=1,2,3,4$ are bounded functions in $L_{\omega}^{2}(Q)$. So for a suitable increasing sequence of integers $n_{k}, k=1,2, \ldots$, there exist functions

$$
u, v \text { and } v_{j}, j=1,2,3,4
$$


in $L_{\omega}^{2}(Q)$ such that

$$
\widetilde{u_{n_{k}}} \rightarrow u, \widetilde{\partial_{t} u_{n_{k}}} \rightarrow v, \widetilde{\partial_{x}^{j} u_{n_{k}}} \rightarrow v_{j}, j=1,2,3,4,
$$

weakly in $L_{\omega}^{2}(Q)$, as $k \rightarrow \infty$. Clearly,

$$
v=\partial_{t} u, v_{j}=\partial_{x}^{j} u, j=1,2,3,4,
$$

in the sense of distributions in $Q$. So, $u \in H_{\omega}^{1,4}(Q)$ and

$$
\partial_{t} u+\partial_{x}^{4} u=f \text { in } Q
$$

On the other hand, the solution $u$ satisfies the boundary conditions

$$
\left.\partial_{x} u\right|_{\Gamma_{i}}=\partial_{x}^{3} u+\left.\beta_{i} u\right|_{\Gamma_{i}}=0, i=1,2,
$$

since

$$
\forall n \in \mathbb{N}^{*},\left.\quad u\right|_{Q_{n}}=u_{n} .
$$

This proves the existence of solution to Problem (3). This ends the proof of Theorem 4.1 .

Acknowledgments. I am thankful to the referees for the valuable remarks which led to an improvement of the original manuscript.

\section{References}

[1] Amster P., Jüngel A., Matthes D.B.-K. Non-homogeneous boundary conditions for a fourth-order diffusion equation. C. R. Acad. Sci. Paris, Ser. 2008, (I) (346): 143-148

[2] Baderko E. A. On the solution of the boundary value problems for parabolic equations of high order in domains with curvilinear lateral boundaries. Diff, Urav., 1976, 12(2):1781-1792

[3] Baderko, E. A. On the solution of boundary value problems for linear parabolic equations of arbitrary order in noncylindrical domains by the method of boundary integral equations $[\mathrm{Ph} \mathrm{D}$ Thesis]. Moscow, 1992

[4] Besov V. The Continuation of Function in $L_{p}^{1}$ and $W_{p}^{1}$. Proc. Steklov Inst. Math., 1967, (89):5-17

[5] Cherepova M. F. On the solvability of boundary value problems for a higher order parabolic equation with growing coefficients. Doklady Mathematics, 2006, 74(3):819-820

[6] Cherepova M. F. Regularity of solutions of boundary value problems for a second-order parabolic equation in weighted Hölder spaces. Differential Equations, 2013, 1 (49):79-87

[7] Galaktionov V. A. On regularity of boundary point for higer-order parabolic equations: towards Petrovskii-type criterion by blow-up approach, Nonlinear Differential Equations and Applications, 2009, (16):597-655

[8] P. Grisvard, Singularities in Boundary Values Problems, Masson, Paris, 1992

[9] Kheloufi, A. Study of the first boundary value problem for a fourth order parabolic equation in a nonregular domain of $\mathbb{R}^{N+1}$, TWMS J. App. Eng. Math., 2015, 1(5): 145-157

[10] Kheloufi, A. On a fourth order parabolic equation in a nonregular domain of $\mathbb{R}^{3}$. Mediterr. J. Math. 2015, (12): 803-820

[11] Kondratiev, V.A. Boundary value problems for parabolic equations in closed regions. Trans. Moscow Math. Soc., vol. 15, Am. Math. Soc., Providence, RI, 1966, pp. 450-504

[12] Kondratiev, V.A.: Boundary value problems for elliptic equations in domains with conical or angular points. Trans. Moscow Math. Soc., vol. 16,Am.Math.Soc., Providence, RI, 1967, pp. $227-313$ 
[13] Labbas, R., Sadallah, B.-K.: Smoothness of the solution of a fourth order parabolic equation in a polygonal domain. Int. J. Appl. Math. 1999, (1):75-90

[14] Ladyzhenskaya O. A., Solonnikov V. A., Ural'tseva N. N. Linear and Quasi-Linear Equations of Parabolic Type. A.M.S., providence, Rhode Island, 1968

[15] Lions J. L., Magenes E. Problèmes aux Limites Non Homogènes et Applications, 1, 2. Dunod, Paris, 1968

[16] Mikhailov, V.P. The Dirichlet problem for a parabolic equation, I. Mat. Sb. (N.S.) 61(103), 40-64 (1963)

[17] Mikhailov, V.P. The Dirichlet problem for a parabolic equation, II. Mat. Sb. (N.S.), 1963, 62(104): 140-159

[18] Sadallah B.-K. Etude d'un problème 2m-parabolique dans des domaines plan non rectangulaires. Boll. Un. Mat. Ital., 1983, 2-B(5):51-112

[19] Sadallah B.-K. High order regularity of the singularities of the solution of a parabolic equation in a singular domain, Kuwait Journal of Sciences and Engineering, 1997, (24): 185-198

[20] Sadallah B.-K. Singularities of the solution of a $2 \mathrm{~m}$-parabolic problem in a polygonal domain, Arab Journal of Mathematical Sciences, 1998, 2(4):31-41 